\title{
Experimental Study on \\ a Prism-External-Cavity Semiconductor Laser with Dispersion Feedback
}

\author{
Guang XU, Kan-ichi FUJII, and Shigeru NAKAYAMA* \\ Department of Electrical and Electronic Engineering, Faculty of Engineering, Ibaraki University \\ 4-12-1 Nakanarusawa-machi, Hitachi Ibaraki 316 \\ *Quantum Electronics Laboratory, Hyogo University of Teacher Education \\ 942-1 Shimokume, Yashiro-cho, Kato Hyogo 673-14
}

(Received September 26, 1996)

\begin{abstract}
We have studied experimentally a prism-external-cavity semiconductor laser system with a dispersion feedback. In the system, the longitudinal mode of a semiconductor laser can be tuned continuously over a small range of about $400 \mathrm{MHz}$, and the linewidth of a single mode is about $60 \mathrm{MHz}$. Tuning characteristics have been investigated as functions of injection current, external cavity length and feedback energy to the laser diode.
\end{abstract}

Key Words: Semiconductor laser, External cavity, Dispersion feedback, Single mode tuning

\section{Introduction}

In many cases of semiconductor laser systems, a beam reflected back into semiconductor laser from optical elements is a cause of noise or fluctuation in both output power and oscillating frequency. This becomes a bottleneck in its applications. However, the operation of a semiconductor laser can be improved by controlling the feedback energy and phase with an additional optical mirror. This is called an external cavity semiconductor laser, and has received much attention ${ }^{1-4)}$ in recent years. It is promising technique for a variety of applications, for instance, cell biology where high resolution spectroscopy might offer new diagnostic methods.

A diffraction feedback system in which a grating is used as the frequency selecting element is well known as the external cavity semiconductor laser. Zorabedian ${ }^{3)}$ developed a gratingexternal-cavity semiconductor laser containing prisms. The prisms were only used as beam expanders between the laser and the grating.

The dispersion feedback system has some advantage in comparison with the diffraction feedback system. First, optical power can be fed back more effectively in the dispersion feedback system than in the diffraction feedback system, because the feedback beam is transmitted light in the dispersion feedback system but the first order of diffracted light in the diffraction feedback system. Second, it is easy in the dispersion feedback system to control the feedback power by changing the reflectivity of the external mirror or by inserting the ND filter between the mirror and the prism.

In this study, we have employed a prism as the frequency selecting element and studied experimentally a prism-externalcavity semiconductor laser with a dispersion feedback system. Even in the dispersion feedback system, it became possible to tune the longitudinal mode continuously over a range of about $400 \mathrm{MHz}$.

\section{Cavity design}

In this work, the semiconductor laser was driven by a couple of large capacity rechargeable solid state batteries in order to eliminate the AC noise of the commercial network and to obtain a stable longitudinal mode of semiconductor laser oscillation. The output spectra were analyzed with a scanning confocal Fabry-Perot interferometer (Technical Optics Ltd., SA-7.5) which has a free spectral range (FSR) of $7.5 \mathrm{GHz}$ and a Finesse of 175. The obtained spectra were displayed on an oscilloscope and stored on a floppy disk with a personal computer.

The configuration of the dispersion feedback system is shown in Fig.1. The semiconductor laser used is an AlGaInP multiquantum-well single longitudinal mode laser type, HL6312G made by Hitachi Ltd.. It has emission wavelength of $630 \mathrm{~nm}$ with output power of $5 \mathrm{~mW}$ for $\mathrm{CW}$ operation. Output of the semiconductor laser is dispersed by a prism and partially reflected by the surface of the prism, after collimation by a lens that has an effective diameter of $3.3 \mathrm{~mm}$ and a numerical aperture of 0.45 . The prism used is a $60^{\circ}$ silica dispersion prism. The dispersed beam is transmitted through a neutral density (ND) filter and reflected back into the laser by a mirror mounted on a piezo transducer (PZT, Sigma Koki Ltd., ASM-20). The feedback energy to the semiconductor laser was controlled by changing the transmission of the neutral density filter. The partially reflected beam from the surface of the prism was used as the frequency tuned output.

Frequency tuning should be achieved through control of both the mirror angle and the mirror translation by changing the voltage applied to the PZT. However, we could not change the mir- 


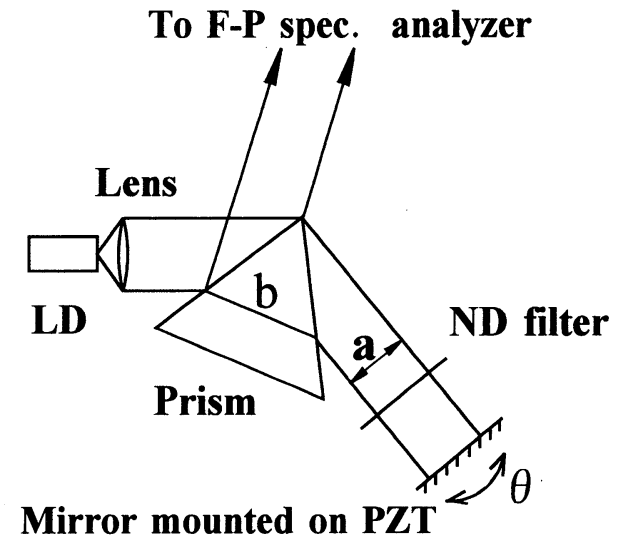

Fig.1 Schematic diagram of the external cavity semiconductor laser using a prism as the frequency-tuning element.

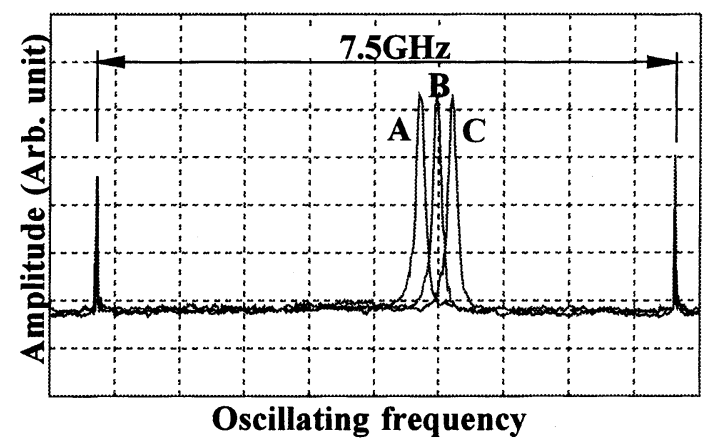

Fig.2 Tuning characteristics of the dispersion feedback system. By varying the potential applied to the PZT, the longitudinal mode was tuned continuously from A to $\mathrm{C}$.

ror translation because of mechanical malfunction of the piezo transducer. In our preliminary experiment, we could control only the mirror angle.

\section{Experimental results and considerations}

3.1 Tuning characteristics of the dispersion feedback system Tuning characteristics of the dispersion feedback system are shown in Fig.2. In Fig.2, the tuning range of the longitudinal mode is several hundreds of $\mathrm{MHz}$ in the same mode. The tuning range of the single mode is limited by the appearance of side modes and mode hopping.

The tuning characteristics of the dispersion feedback system can be explained as follows: Output of the laser will be dispersed by the prism. The dispersed beams with different wavelength (or frequency) will have different direction of propagation which are determined by the following equation: ${ }^{5)}$

$$
\delta \varepsilon=\frac{b}{a} \frac{\mathrm{d} n}{\mathrm{~d} \lambda} \delta \lambda
$$

where $\delta \varepsilon$ is the dispersion angle between the directions of beams with wavelengths between $\lambda$ and $\lambda+\delta \lambda, a$ is the diameter of beam, $b$ is the geometrical length shown in Fig.1.

It can be seen that if the external mirror angle is controlled, a beam with a different frequency will be reflected back into the semiconductor laser (LD in Fig.1) and begin to oscillate in the compound cavity, due to the broad gain spectrum of the semiconductor laser.

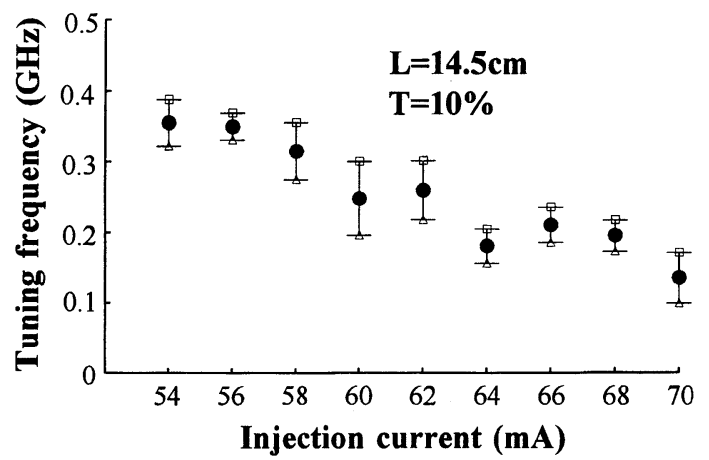

Fig.3 Change of the tuning frequency as a function of the injection current in the dispersion feedback system.

3.2 Dependence of the injection current on the tuning characteristics

Figure 3 shows the tuning range of frequency as a function of injection current. The length $L$ of external cavity $(14.5 \mathrm{~cm})$, and the transmittance $T$ of the ND filter (10\%) were fixed, while the injection current $i$ was varied.

In this experiment, frequency tuning was successful although the tuning range decreased with increasing of the injection current. This is interpreted as follows: At a high injection level, the mode of the laser is highly self-organized, thereby the feedback beam exhibits a small effects on the internal mode. On the condition that the injection current is smaller than $54 \mathrm{~mA}$, the output of the system was too weak to analyze the modes, while if the injection current is larger than $70 \mathrm{~mA}$, the tuning range is too narrow to analyze.

3.3 Dependence of the feedback energy on the tuning characteristics

Figure 4 shows the tuning range of frequency as a function of transmittance of the ND filter, therefore the feedback energy. The length $L$ of the external cavity $(14.5 \mathrm{~cm})$ and the injection current $i(54 \mathrm{~mA})$ were fixed, while the feedback energy was varied by changing the transmittance $T$ of the ND filter.

In the region of transmittance of the ND filter smaller than $5 \%$, the tuning range of frequency increases with the increase of the transmittance, i.e., the increase of the feedback energy. In the case of the weak feedback, the feedback beam hardly interferes with semiconductor laser. Therefore, the tunability increases with increasing the feedback energy. In the region of transmittance greater than 5\%, the frequency tuning range of the longitudinal mode was saturated. When the transmittance of the filter is greater than $10 \%$, the longitudinal mode became unstable and noisy. That is, mode instability appears by the socalled "coherence collapse" due to instabilities in the output intensity. ${ }^{6)}$

In this experiment, it was rather difficult to determine the exact amount of external feedback energy, because the radius of the feedback beam at the front facet of the laser diode becomes larger than the size of the active layer due to the diffraction effect, and existence of other coupling losses like the reflection from the facet of the laser.

3.4 Dependence of the cavity length on the tuning characteristics

Figure 5 shows the frequency tuning range as a function of cavity length. The feedback energy (transmittance $T$ of 10\%) 


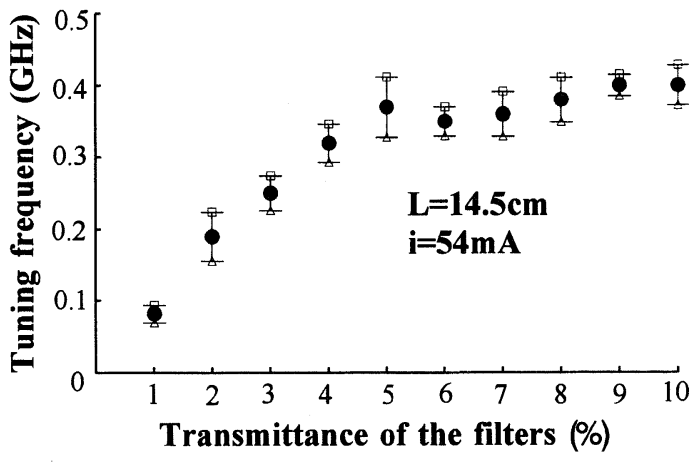

Fig.4 Change of tuning frequency as a function of transmittance of the ND filter in the dispersion feedback system.

and the injection current $i(54 \mathrm{~mA})$ were fixed, while the length $L$ of the external cavity was varied.

The frequency tuning was successful in the cavity length from 14.5 to $17.5 \mathrm{~cm}$. The shorter the cavity length, the larger the tuning frequency region is. However, the shortest cavity length was limited to $14.5 \mathrm{~cm}$ for the convenience of setting up this experiment. For the cavity length longer than $17.5 \mathrm{~cm}$, the longitudinal mode became unstable and noisy.

This fact can be interpreted as follows: When the cavity length becomes longer, the mode spacing of the intercavity becomes narrower. Since the gain profile remains unchanged, several modes could begin to oscillate at the same time. This will disturb the single mode oscillation in the upper limit $17.5 \mathrm{~cm}$ of the cavity length. The frequency tuning should be achieved through control of both the mirror angle and the mirror translation. However, we could not control the mirror translation because of the mechanical malfunction of the piezo transducer. Therefore, we could not tune the wide range over the mode spacing of the intercavity. This will result in the lower limit $14.5 \mathrm{~cm}$ of the cavity length.

The oscillation frequency of a semiconductor laser strongly depends on the refractive index of the semiconductor which is affected by the fluctuation of the temperature of the semiconductor material, the injection current, therefore, the excited carrier density in the conduction band, and so on. To overcome these shortcomings, and to keep the oscillating frequency constant, such factors must be fixed at constant value. Therefore, we can decouple the resonant frequency from the strong dependence on the refractive index of the semiconductor by introducing the external cavity method.

Since the optical length of the external cavity is $n l+L$, the frequencies $v_{\mathrm{m}}$ of the modes can be written as:

$$
v_{\mathrm{m}}=\frac{m c}{2(n l+L)}, \quad m=1,2 \cdots
$$

where $n$ is the refractive index of the medium, $l$ is the length of the diode, $L$ is the optical length of the external cavity, $c$ is the speed of light, and $m$ is an integer. Differentiating equation (2) by refractive index, we obtain:

$$
\frac{1}{v_{\mathrm{m}}} \frac{\partial v_{\mathrm{m}}}{\partial n}=\frac{1}{n} \frac{-1}{(L / n l+1)}
$$

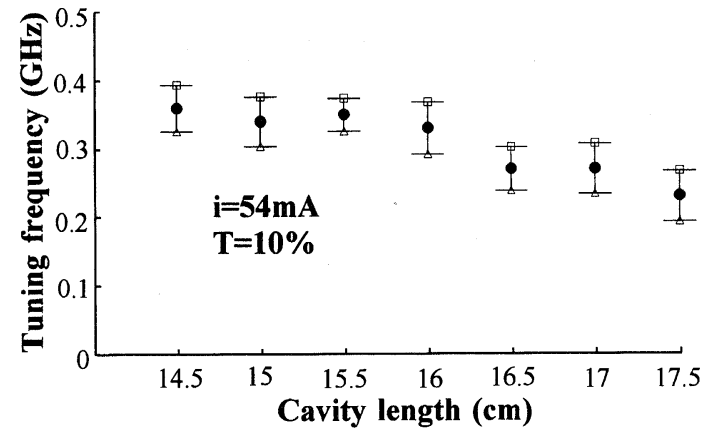

Fig.5 Change of the tuning frequency as a function of the length of the external cavity in the dispersion feedback system.

For $L \gg n l$, the relative change in the mode frequency due to the change in the refractive index are reduced by the factor of $n l / L$ compared with a semiconductor laser without external cavity. Thus the external cavity semiconductor laser has a better stability of oscillation frequency than that of the solitary semiconductor laser.

\section{Conclusions}

We have tried to construct the prism-external-cavity semiconductor laser system with dispersion feedback and compared the characteristics with those of the grating-external-cavity semiconductor laser system with diffraction feedback. In the dispersion feedback system, the longitudinal mode can be tuned continuously over a small range of about $400 \mathrm{MHz}$, and the linewidth of a single mode is about $60 \mathrm{MHz}$. In order to obtain a wider frequency tunability, it is necessary to control the mirror translation and it is desirable to use a small external cavity length, moderate feedback power, and relatively small injection current.

These results can be applied to high resolution laser spectroscopy as well as to a compact, and less expensive fluorescence cell sorter (FACS) for cell biology applications.

\section{Acknowledgements}

The authors are grateful to Ministry of Education, Science and Culture for an International Joint Research (1994 1996) Grant (No. 06044027).

\section{References}

1) R. Lang and K. Kobayashi: IEEE J. Quantum. Electron. QE-16 (1980) 347.

2) H. Kakiuchida and J. Ohtsubo: IEEE J. Quantum. Electron. QE-30 (1994) 2087.

3) P. Zorabedian: IEEE J. Lightwave Technology LW-10 (1992) 330.

4) P. Besnard, B. Meziane, and G. M. Stephan: IEEE J. Quantum. Electron. QE-29 (1993) 1271.

5) M. Born and E. Wolf: Principles of Optics (Pergamon Press, Oxford, 1974).

6) D. Lenstra, B. H. Verbeek, and A. J. Denoef: IEEE J. Quantum. Electron. QE-21 (1985) 674. 